\title{
1 REVA as a Well-curated Database for Human Expression-modulating
}

\section{Variants}

3

4 Yu Wang ${ }^{1, \#}$, Fang-Yuan Shi ${ }^{1, \#}$, Yu Liang ${ }^{2}$, Ge Gao ${ }^{1, *}$

5

$6 \quad{ }^{1}$ Biomedical Pioneering Innovation Center \& Beijing Advanced Innovation Center for Genomics,

7 Center for Bioinformatics and State Key Laboratory of Protein and Plant Gene Research, School of

8 Life Sciences, Peking University, Beijing 100871, China.

$9{ }^{2}$ Human Aging Research Institute, School of Life Sciences, Nanchang University, Nanchang 10 330031, China.

11

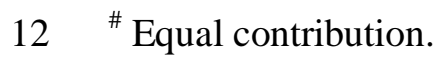

$13{ }^{*}$ Corresponding author.

14 E-mail: gaog@mail.cbi.pku.edu.cn (Gao G).

15

16 Running title: Wang Y et al / REVA: Expression-modulating Variants Database

18 Total word counts: 3540

19 Total references: 57

20 Total tables: 2

21 Total figures: 4

22 Total supplementary figures: 6

23 Total supplementary tables: 9

24 Counts of letters in article title: 64

25 Counts of letters in running title: 42

26 Counts of keywords: 5

27 Counts of words in abstract: 99 


\section{Abstract}

2 More than $80 \%$ of disease- and trait-associated human variants are noncoding. By

3 systematically screening multiple large-scale studies, we compiled REVA, a manually

4 curated database for over 11.8 million experimentally tested noncoding variants with

5 expression-modulating potentials. We provided 2424 functional annotations that could

6 be used to pinpoint plausible regulatory mechanism of these variants. We further

7 benchmarked multiple state-of-the-art computational tools and found their limited

8 sensitivity remains a serious challenge for effective large-scale analysis. REVA

9 provides high-qualify experimentally tested expression-modulating variants with

10 extensive functional annotations, which will be useful for users in the noncoding

11 variants community. REVA is available at http://reva.gao-lab.org.

12

13 KEYWORDS: Noncoding variants; Expression-modulating variants; Massively

14 parallel reporter assay; Database; Benchmark

15 


\section{Introduction}

2 Noncoding regions occupy the majority of the human genome [1]. It has been

3 demonstrated that noncoding variants can affect the regulation of genes [2] and more

4 than $80 \%$ of disease- and trait-associated variants are noncoding variants [3].

5 Noncoding variants that could affect gene expression can be considered as

6 expression-modulating variants [4]. Several experimental assays have been developed

7 to characterize expression-modulating variants. Genome editing technologies such as

8 transcription activator-like effector nucleases (TALENs), zinc finger nucleases

9 (ZFNs), and clustered regularly interspaced short palindromic repeats with Cas9

10 nuclease (CRISPR/Cas9) provide high-quality validated data but are generally low

11 throughput [5-7]. Recently developed massively parallel reporter assays (MPRAs)

12 can identify transcriptional regulatory elements in an efficient way, allowing

13 systematic screening of tens of thousands of genetic variants for pinpointing the

14 causal variants of complex traits $[4,8,9]$. All expression-modulating variants stored

15 in MaveDB [10] are validated by the MPRA experiments. MPRAs have generated

16 over 10 million human expression-modulating variants [11], however, only around 30

17 thousand of them have been collected by MaveDB without any functional annotation,

18 which hinders the further utilization of these data.

19 Though experimental assays for characterizing noncoding 20 expression-modulating variants have generated huge amount of data, it is still 21 inadequate for covering all noncoding variants identified in human genomes. 22 Therefore, multiple computational tools have been developed for identifying 23 expression-modulating variants (Table 1). Transcription factors (TFs) could regulate 24 genes through binding to sequence motifs [12] and noncoding variants could affect gene regulation by changing motifs [13]. Funseq2 integrated a module for detecting motif-breaking and -gain events through the change of position weight matrix (PWM)

27 and other functional annotations to prioritizing cancer driver mutations [14]. Methods

28 based on machine learning have been used wildly in biological researches [15]. 29 CADD [16] used support vector machine (SVM) to classify variants into functional 30 and nonfunctional variants and GWAVA [17] used random forest to predict 31 disease-related variants. Both of CADD and GVAWA are based on supervised 32 learning methods, while Eigen [18] implemented unsupervised learning methods to 33 classify variants. All these tools highly depend on existing annotations at 
1 corresponding loci. In 2015, Alipanahi et al. [19] developed DeepBind based on 2 convolutional neural networks (CNNs) to predict the binding affinity between TFs 3 and DNA or RNA binding proteins and RNA. DeepSEA [20] applied similar methods

4 to predict the effect of noncoding variants on binding affinity and then classify 5 variants through logistic regression into functional or nonfunctional groups. All tools 6 mentioned above identifying expression-modulating variants are through indirect 7 inference, because they are not trained on expression-modulating variants or 8 expression-related data. EnsembleExpr [21] used MPAR data for training an 9 ensemble-based model to characterize expression-modulating variants directly. 10 ExPecto [22] ab initio predicts the variants' effects on gene expression from $40 \mathrm{~kb}$ 11 promoter-proximal sequences and then pinpoints expression-modulating variants. 12 However, there is no comprehensive evaluation of these computational tools based on 13 high-quality expression-modulating variants, therefore, it is difficult for users to 14 choose appropriate tools for their tasks.

15 Here, we present a repository for expression-modulating variants (REVA). The 16 current release of REVA consists of over 11.8 million experimentally validated 17 expression-modulating variants in the human genome, curated with extensive 18 functional annotations. We further benchmarked seven popular computational tools in 19 identifying expression-modulating variants [14, 16-18, 20-22] based on high-quality 20 data in REVA. All data and benchmarking results are publicly available at 21 http://reva.gao-lab.org. 


\section{Construction and content}

\section{Data collection and integration}

3 To ensure unified and high-quality data, all records in REVA were collected and

4 curated using a standard procedure (Figure 1). We used a list of keywords, "MRPA",

5 "STARR-seq", "CRE-seq" with "mutation", "variant" and "variation", to obtain

6 publications from PubMed (https://pubmed.ncbi.nlm.nih.gov/) and then manually

7 checked the abstracts and full texts of the matching publications to obtain literatures

8 that experimentally validated the effects of noncoding expression-modulating

9 variants.

For filtered literatures, we extracted related information of the variants from the

11 main text as well as supplementary materials of publications and converted them to 12 the same format (Table 2). Variants that failed to be mapped to both GRCh37 and 13 GRCh38 were removed. Variants only mapped to coding region were also removed. 14 For missing information, we used "." as a placeholder. In addition, the detail protocol and raw data of the experiments were also extracted.

For variant data with the same chromosome, genome location, reference allele, alternative allele and experimental cell line from different publications, a meta-analysis was conducted to integrate data. The harmonic mean p-value (HMP) method [23] was used in the meta-analysis, and the cutoff for the meta p-value was set to 0.001 to generate the meta-label. The variants involved in the meta-analysis but without a raw p-value were also removed.

22 The label of variants was given based on the cutoff for the adjusted p-value or 23 meta $\mathrm{p}$-value, and then variants were classified into positive variants and negative variants based on label or meta-label. If the variant's label was 1, the variant was a positive variant and considered to have effects on gene expression, otherwise, it was a negative variant without effect on gene expression.

\section{Database construction}

28 All manually curated variant data as well as meta-information were stored in 29 MongoDB (https://www.mongodb.com/) at two levels: accession and variant data 30 (Supplementary Figure S1). Each accession entry consisted of an accession number, 31 the times of creation and last update for the accession, information about the assay 32 used in the publication (method type, original reference genome version, link to raw data, and summary of the assay), and the reference. Variant data include all related 
1 information about the variant, and each variant data entry is linked to one accession.

2 For the data involved in the meta-analysis, the variant data contained the results of the

3 meta-analysis and linked to all related variants and accessions.

4

We also integrated DisGeNET v7.0 (https://www.disgenet.org/) variant-disease associations, GWAS catalog (https://www.ebi.ac.uk/gwas/), ClinVar

6 (https://www.ncbi.nlm.nih.gov/clinvar/), COSMIC

7 (https://cancer.sanger.ac.uk/cosmic), and three-dimensional interacting genes and 8 chromatin state from 3DSNP [25] to our database for providing more variant 9 information.

\section{Variant annotation}

11 In efforts to pinpoint plausible regulatory mechanisms for these variants, we used 2403 trained CNNs to annotate the functional effects of sequence variations [26] based on 1249 TF binding, 766 histone modification, 280 DNA accessibility, and 108 14 DNA methylation profiles from recent Encyclopedia of DNA Elements (ENCODE) 15 data.

An NVIDIA Tesla P100 Graphics Processing Unit with the implementation on the deep learning framework TensorFlow (https://www.tensorflow.org/) and Python (https://www.python.org/) were used for training models. We adopted stochastic gradient descent (SGD) as the optimizer and the initial learning rate was 0.01 .

The final output layer of CNN model was a fully connected layer with sigmoid function used to scale the output between 0 to 1 . The input layer was one dimensional convolution layer with threshold ReLU as the activation function. Next, the max pooling layer was performed to reduce the complexity of data. Then dropout layer was used to avoid overfitting problem. The next two layers were full connected layer with threshold ReLU as the activation function and dropout layer.

For TF binding, histone modification, and DNA accessibility models, the positive data for training CNNs were the 200bp sequences centered on the peak in ENCODE profiles. Then we remove positive sequences from human reference genome and split the rest to 200bp bins. Random sampled 200bp bins with the same number of positive data were used as negative data. For DNA methylation, the 200bp sequences centered on the target base with the methylation rate more than 0.5 or less than 0.5 in whole-genome bisulfite sequencing (WGBS) data were considered as positive data

33 and negative data respectively. One-hot encoding was conducted to transformed each 34 sequence to $200 \times 4$ binary matrix for model training. 
1 Five-fold cross-validation strategy was used to train models. During each 2 iteration of model training, $15 \%$ of the input data were random selected as the 3 independent testing dataset to evaluate model performance. The remained data were 4 split with $70 \%$ to train models and $15 \%$ as the validation dataset to optimize 5 parameters. Model performance was evaluated with area under the receiver operating 6 characteristic curve (AUROC) and area under the precision recall curve (AUPRC) to 7 test the sensitivity and specificity, and models with best performance were selected 8 for variant annotation. An average AUROC and AUPRC of 2403 models were 9 reported.

10 To character the binding affinity changes of the variant, we used 2403 trained 11 CNNs to predict on 200bp sequences centered with the reference allele and alternative 12 allele respectively. For each chromatin profiles, the log2-fold-changes (as the method 13 shown in DeepSEA) [20] was calculated as the variant effect on chromatin profiles. 14 Specifically,

$$
\text { Effect }=\log 2\left(\frac{\mathrm{P}_{\mathrm{r}}}{1-\mathrm{P}_{\mathrm{r}}}\right)-\log 2\left(\frac{\mathrm{P}_{\mathrm{a}}}{1-\mathrm{P}_{\mathrm{a}}}\right)
$$

where $\mathrm{P}_{\mathrm{r}}$ is the prediction of sequence with reference allele and $\mathrm{P}_{\mathrm{a}}$ is the prediction of sequence with alternative allele.

17 Furthermore, we incorporated 13 DNA physicochemical properties and 8 18 evolutionary features into the annotation pipeline. The 13 physicochemical properties 19 were calculated as the Rong Li et al. [27] described and 8 conservation scores were 20 downloaded from UCSC Genome Browser (http://genome.ucsc.edu/).

\section{Benchmarking}

22 To prepare the benchmarking dataset for evaluating the performance of 23 state-of-the-art computational tools in calling expression-modulating variants based 24 on the curated data in REVA, we first excluded loci tested in mice $(15,152)$. There are 25 several overlapped variants between the training sets of state-of-the-art tools and the 26 REVA benchmark dataset. If the benchmark dataset contains these variants, the 27 performance of related tools will be overestimated. To avoid the influence of these 28 variants and make a fair comparison, we further removed variants $(47,518)$ that were 29 either found in the GWAVA [17] and EnsembleExpr [21] training datasets or used to 30 compute the empirical background distributions by DeepSEA [20]. For the remaining $315,809,991$ loci (37,816 positive and 5,772,175 negative), we ran CADD (v1.4, 32

[16],

DeepSEA 
1 (http://deepsea.princeton.edu/),

EnsembleExpr

2 (https://github.com/gifford-lab/EnsembleExpr/),

and

ExPecto

3 (https://hb.flatironinstitute.org/expecto/) [22] and extracted from the precomputed

4 score set of Eigen (v1.1, http://www.columbia.edu/ ii2135/download.html) [18],

5 FunSeq2 (v2.1.6, http://funseq2.gersteinlab.org/downloads) [14], and GWAVA

6 (https://www.sanger.ac.uk/science/tools/gwava) to obtain the corresponding predicted

7 score for evaluation. The thresholds used in the evaluation were those recommended

8 by the corresponding papers or official websites (Supplementary Table S1).

9 All variants in benchmark dataset were variants with expression-modulating

10 potential. One of biological mechanisms that diseases-related or phenotype-related

11 variants function is by having effects on gene expression regulation [28]. Pinpointing

12 disease-related or phenotype-related variants is more useful for biomedical researches.

13 Therefore, we further selected the GWAS, ClinVar, and HGMD subsets of benchmark

14 dataset to test these tools' power. 


\section{$1 \quad$ Results}

\section{Characterization and distribution of expression-modulating variants}

3 All curated expression-modulating variants were validated by experiments and we

4 applied standard data collection and integration procedure to ensure the high-quality 5 data with unified format. By the end of November 2019, REVA consisted of

$6 \quad 11,862,367$ entries covering 5,948,789 experimentally tested noncoding loci across 18 7 cell cultures from 14 publications [4, 8, 11, 29-39]. We first excluded loci tested in 8 mice $(15,152)$ and with more than one alternative allele (26,326). Among the 9 remaining 5,907,311 loci (34,700 positive and 5,872,661 negative), most were located 10 in intergenic (positive: 49.96\%, negative: 53.83\%) and intronic (positive: $35.96 \%$, 11 negative: $39.62 \%$ ) regions (Figure 2A, Supplementary Table S2). We found that both 12 positive and negative variants were unevenly distributed on chromosomes, and no 13 variants were located on the Y chromosome (Figure 2B, Supplementary Table S3). 14 Specifically, fewer positive variants were located on chromosomes 1, 3, 5, 13, 14, 15, 1521 and the $\mathrm{X}$ chromosome, and more positive variants were located on chromosomes $6,8,10,11,12$, and 16-20. Fewer negative variants were located on chromosomes 9, $1713,14,15,21,22$ and the $\mathrm{X}$ chromosome, and more negative variants were located on chromosomes 1-8, 10, 11, 12, and 16-20. Biochemical activities were detected for $1993.53 \%$ positive and $90.80 \%$ negative cases in at least one cell culture 20 (Supplementary Figure S2, Supplementary Table S4, S5). Of note, more positive than 21 negative variants were found in TF binding regions, highlighting the contribution of 22 TF binding changes to expression modulation (Supplementary Figure S2).

\section{Extensive functional annotation of expression-modulating variants}

24 We used 2403 trained $\mathrm{CNNs}$ to annotate the functional effects of expression-modulating variants [26]. Most of the trained CNN networks were accurate, with an average AUROC of 0.908 and an average AUPRC of 0.904. Among the 5,789,688 variants annotated, both positive and negative variants were found to lead to significant changes in binding affinity for 22 and 12 TFs on average, respectively, which also suggested that expression-modulating variants may affect

30 gene expression regulation through changing the binding affinity of TFs. Moreover, $318.72 \%$ of positive variants and $3.56 \%$ of negative variants were located at evolutionary conserved loci (phastCons100way score > 0.6). 
1 To evaluate the power of state-of-the-art computational tools in calling 2 expression-modulating variants, we further benchmarked multiple state-of-the-art 3 computational tools based on the curated data in REVA. With the benchmarking 4 dataset containing 5,809,991 loci (37,816 positive and 5,772,175 negative), we found 5 that 1289 could not be predicted by DeepSEA (since their evolutionary features were 6 not available) and 560,577 were not included in the precomputed score set of Eigen, 7 FunSeq2, and GWAVA, so we further excluded these 561,866 cases from follow-up analysis. Meanwhile, as EnsembleExpr could not finish the whole benchmarking dataset in a reasonable amount of time, we assessed its performance based on the

10 average metrics over five randomly sampled subdatasets with 368 positive and 56,026 11 negative cases on average.

12 Overall, the best-performing tool was DeepSEA, with the highest AUROC and 13 F1 score (Figure 3A and B, Supplementary Table S6). All tools performed well in 14 terms of specificity but poorly in terms of sensitivity. EnsembleExpr had the highest sensitivity but the lowest specificity, whereas ExPecto showed the best specificity and worst sensitivity.

17 There were 52,672 variants in the benchmarking dataset that overlapped with the 18 GWAS catalog (v1.0.2), and 658 of them were positive variants. All tools performed 19 better on variants overlapping with the GWAS catalog, and DeepSEA still had the 20 best performance (Figure 3C). Meanwhile, ExPecto and FunSeq2 showed better performance on variants at evolutionarily conserved loci, while DeepSEA had poorer performance (Figure 3D).

The coverage and quality of training data may contribute significantly to the performance of machine learning-based models [40]. To test whether variants from different cell lines would affect the performance of these tools, we further evaluated these tools separately on seven cell lines (Supplementary Table S7). On GM18507, GWAVA-Unmatch performed best; on HEK293T and NA12878_NA19239, Eigen-PC had the highest F1 score; DeepSEA had the best performance on HepG2, K562, and K562_GATA1; and CADD performed best on SH-SY5H (Figure 3E), which suggested that the diversity of the original training data contributed to the performance differences of these tools. Of note, thus far, only ExPecto outputs cell-type-specific scores for various tissues.

To give further explanation of the potential mechanisms of disease-related 34 variants, we evaluated the benchmarking dataset on disease-related variants. There 
1 were 1400 variants in the benchmarking dataset that overlapped with HGMD (2019.3

2 professional) and 69 of them were positive variants (Supplementary Table S8). 8 of 69

3 variants were verified to regulated gene expression by independent experiments. 40 of

469 variants were associated with diseases such as colorectal cancer, nervous system

5 diseases, and autoimmune diseases. To test computational tools' power on

6 disease-related variants, we compared their performance on these variants. All tools

7 performed better on HGMD variants and DeepSEA still had the best performance

8 (Figure 3F), same on variants with class "DM?" and "DFP". Eigen-PC showed better

9 performance on variants with class "DP". Interestingly, ExPecto performed best on

10 variants with class "FP" but worst on variants with other classes. We also evaluated

11 on variants overlapped with ClinVar (2019.10.08), and DeepSEA had the best overall

12 performance and Eigen showed better performance on "Drug response" related

13 variants (Supplementary Figure S3, Supplementary Table S9).

14 Web interface

15 REVA (http://reva.gao-lab.org) provides an interactive Web interface for users to 16 explore all data entries and analysis results (Figure 4, Supplementary Figure S4).

17 Users can start a quick search by chromosome position, rs id, gene name, ensembl 18 gene id, or disease name. "Advanced search" provides a customized search and batch 19 search for users. The query result is presented as a table, which includes basic 20 information, expression information (such as the label, effect size and adjusted $\mathrm{p}$ 21 value), and the related genomic region. Users can directly click the link of position 22 and rs id to access UCSC Genome Browser and dbSNP 23 (https://www.ncbi.nlm.nih.gov/snp/) for more information. Users can also click the 24 "details" link for more information. The detail page contains eight modules: "Basic 25 information", "Cell Line and Expression", "Three-dimensional Interacting Gene", 26 "Chromatin State", "Disease and Phenotype", "Meta Sources" (only available for 27 variants involved in meta-analysis), "Accession", and "Annotation". In the 28 "Annotation" module, chromatin profile features are rendered as a heatmap by cell 29 line and a boxplot by category, and DNA physicochemical properties and 30 evolutionary features are presented as responsive tables. Users can download the 31 annotation for further analysis. Moreover, we also provide benchmarking results of 32 state-of-the-art computational tools. Users can download all variants in REVA and the 33 benchmarking dataset through the "Download" page.

34 Explore plausible regulatory mechanisms of expression-modulating variants 
1 Autoimmune diseases are caused by abnormal immune response to attack and damage 2 functional tissues due to complex interactions between environmental and genetic 3 factors [41]. GWAS and fine mapping studies have identified thousands of noncoding 4 variants associated with autoimmune diseases [42]. Since the mechanism of 5 autoimmune disease is complicated, pinpointing causal variants and exploring their 6 possible functional mechanism remain a challenge [43].

7 Ankylosing spondylitis is a kind of chronic autoimmune disease but the 8 pathogenesis remains unclear [44]. On the advanced search page of REVA 9 (Supplementary Figure S5), we filtered label to positive and search with "ankylosing 10 spondylitis". The search result contained 8 entries and among the result, the variant 11 rs4456788 (near ICOSLG locus) had the largest effect size tested in HepG2 cell line 12 and considered to repress expression. It was also tested in K562 cell line and resulting 13 in same conclusion. Through the annotation module of the detail page, we found that 14 in HepG2 cell line, the alternative allele of rs4456788 could decrease the binding 15 affinity of transcription factor MAZ and FUS. MAZ has been proven to have 16 bidirectional transcriptional regulation [45] and FUS has transcriptional activation 17 function [46]. It could be the possible regulatory mechanism of rs445678, and this 18 might be helpful for further researches on the mechanism of pathogenesis of 19 ankylosing spondylitis. 


\section{Discussion}

2 REVA is a database specifically designed for storing experimentally validated 3 expression-modulating data. It currently consists of 11,862,367 entries covering $45,948,789$ experimentally tested noncoding loci across 18 cell cultures. Both 5 experimentally validated expression-modulating variants and meta-information about 6 assays were curated. Comparing with the existing database, REVA is the largest 7 database designed for curating experimentally validated expression-modulating 8 noncoding variants specially. Besides, we provide 2424 functional annotations 9 including Transcription Factors binding, epigenetic modifications, DNA accessibility, DNA physicochemical properties, and evolutionary features.

Most of variants in REVA were located in intergenic and intronic regions and 12 were unevenly distributed on chromosomes. Several factors may contribute to the 13 uneven distributions. First, it has been well demonstrated that the functional elements 14 are uneven distributed across chromosomes [47, 48]. Consistently, we found that the number of both positive and negative variants are highly correlated with the gene numbers across all chromosomes (Pearson's $r=0.80, p=2.6 \times 10^{-6}$ for positive variants; Pearson's $r=0.82, p=7.1 \times 10^{-7}$ for negative variants). Moreover, technical challenges count too. In particular, the Y chromosome had long been taken as a "genetic wasteland" [49] and excluded from genomic analyses for quite some time,

20 due to its genetic and structure complexity [50]. Though this idea has been shifted 21 with more researches on chromosome $\mathrm{Y}$, the underrepresentation of chromosome $\mathrm{Y}$ on commonly used arrays still exist [51]. We also notice that certain experimental designs may also lead to reporting bias [8, 31, 36-39]. However, after removing data generated from studies designed for assessing particular regions [36] or elements [8, 31, 37-39], we found that uneven distribution remains.

Furthermore, we provide a high-quality benchmarking dataset for evaluating state-of-the-art computational tools designed for identifying expression-modulating variants as well as benchmarking results of multiple published computational tools, as a reference for users to select the best tools for their particular tasks. Overall, all seven

30 tools have high specificity but low sensitivity. DeepSEA has the best performance on 31 whole benchmark dataset in terms of AUROC and F1-score and all tools have better performance on disease-related or phenotype-related variants, suggesting that the 
1 performance across different benchmark subsets. We noticed that not all tools 2 involved in benchmark were designed for identifying expression-modulating variants 3 originally, and a "negative" expression-modulating noncoding variant might also be

4 associated with disease via non-transcription mechanisms like epigenetic marks [52] 5 or chromatin structuration [53].

6 It should be noted that not all variants collected in our database were tested by 7 identical experimental protocols. Non-saturation mutagenesis-based studies examine 8 several elements at a time, and each fragment usually contains one variant, with the 9 effect size calculated by counting reads directly [8] or employing a linear model [30].

10 Meanwhile, saturation mutagenesis-based studies focus on a few elements; each 11 fragment contains two or more variants, and the effect size is calculated through linear 12 regression [39]. Protocol details for each variant were documented during curation to 13 help users interpret records effectively (Supplementary Figure S6).

14 We believe that this database will be useful for not only computational but also 15 bench biologists in genomics, bioinformatics and genetics community, and we will 16 keep the resources updated with new data and annotations emerged in the coming 17 years. 


\section{Data availability}

2 REVA is freely accessible at http://reva.gao-lab.org.

3

4 Code availability

5 Source code for all analysis and benchmark is available on GitHub at

6 https://github.com/gao-lab/REVA-Data Source Code.

\section{$8 \quad$ CRediT author statement}

9 Yu Wang: Methodology, Software, Data Curation, Formal analysis, Visualization,

10 Writing - Original Draft, Writing - Review \& Editing. Fang-Yuan Shi: Methodology,

11 Software, Data Curation, Formal analysis. Yu Liang: Data Curation. Ge Gao:

12 Conceptualization, Project administration, Supervision, Funding acquisition,

13 Resources, Writing - Review \& Editing.

14

\section{Competing interests}

16 The authors declare no competing interests.

\section{Acknowledgments}

19 This work was supported by funds from the National Key Research and Development 20 Program of China (2016YFC0901603), the National High Technology Research and 21 Development Program of China (2015AA020108), as well as the State Key 22 Laboratory of Protein and Plant Gene Research and the Beijing Advanced Innovation 23 Center for Genomics (ICG) at Peking University. The research of Ge Gao was 24 supported in part by the National Program for Support of Top-notch Young 25 Professionals.

26 Part of the analysis was performed on the Computing Platform of the Center for 27 Life Sciences of Peking University and was supported by the High-performance 28 Computing Platform of Peking University.

\section{ORCID}

31 0000-0003-2799-0369 (Yu Wang)

32 0000-0003-4185-8129 (Fang-Yuan Shi) 
bioRxiv preprint doi: https://doi.org/10.1101/2021.02.24.432622; this version posted February 25, 2021. The copyright holder for this preprint (which was not certified by peer review) is the author/funder. All rights reserved. No reuse allowed without permission.

1 0000-0003-0695-2304 (Yu Liang)

2 0000-0001-6470-8815 (Ge Gao)

3 


\section{$1 \quad$ References}

2 [1] Gonzaga-Jauregui C, Lupski JR, Gibbs RA. Human genome sequencing in health and disease. Annu Rev Med 2012;63:35-61.

[2] Gusev A, Lee SH, Trynka G, Finucane H, Vilhjálmsson BJ, Xu H, et al. Partitioning heritability of regulatory and cell-type-specific variants across 11 common diseases. Am J Hum Genet 2014;95:535-52.

[3] Maurano MT, Humbert R, Rynes E, Thurman RE, Haugen E, Wang H, et al. Systematic localization of common disease-associated variation in regulatory DNA. Science 2012;337:1190-5.

[4] Tewhey R, Kotliar D, Park DS, Liu B, Winnicki S, Reilly SK, et al. Direct identification of hundreds of expression-modulating variants using a multiplexed reporter assay. Cell 2016;165:1519-29.

[5] Spisák S, Lawrenson K, Fu Y, Csabai I, Cottman RT, Seo J, et al. CAUSEL: an

[6] Robbez-Masson LJ, Bödör C, Jones JL, Hurst HC, Fitzgibbon J, Hart IR, et al. Functional analysis of a breast cancer-associated FGFR2 single nucleotide polymorphism using zinc finger mediated genome editing. PLoS One 2013;8:e78839.

[7] Claussnitzer M, Dankel SN, Klocke B, Grallert H, Glunk V, Berulava T, et al. Leveraging cross-species transcription factor binding site patterns: from diabetes risk loci to disease mechanisms. Cell 2014;156:343-58.

[8] Melnikov A, Murugan A, Zhang X, Tesileanu T, Wang L, Rogov P, et al. Systematic dissection and optimization of inducible enhancers in human cells using a massively parallel reporter assay. Nat Biotechnol 2012;30:271-7.

[9] Madan N, Ghazi AR, Kong X, Chen ES, Shaw CA, Edelstein LC.

Functionalization of CD36 cardiovascular disease and expression associated variants by interdisciplinary high throughput analysis. PLoS Genet 2019; 15:e1008287.

[10] Esposito D, Weile J, Shendure J, Starita LM, Papenfuss AT, Roth FP, et al. MaveDB: an open-source platform to distribute and interpret data from multiplexed assays of variant effect. Genome Biol 2019;20:223. 
1 Comoglio F, et al. High-throughput identification of human SNPs affecting 2 regulatory element activity. Nat Genet 2019;51:1160-9.

3 [12] Latchman DS. Transcription factors: an overview. Int J Biochem Cell Biol 1997;29:1305-12.

[13] Mu XJ, Lu ZJ, Kong Y, Lam HYK, Gerstein MB. Analysis of genomic variation in non-coding elements using population-scale sequencing data from the 1000 Genomes Project. Nucleic Acids Res 2011;39:7058-76.

[14] Fu Y, Liu Z, Lou S, Bedford J, Mu XJ, Yip KY, et al. FunSeq2: a framework for prioritizing noncoding regulatory variants in cancer. Genome Biol 2014;15:480.

[15] Wainberg M, Merico D, Delong A, Frey BJ. Deep learning in biomedicine. Nat Biotechnol 2018;36:829-38.

[16] Kircher M, Witten DM, Jain P, O'Roak BJ, Cooper GM, Shendure J. A general framework for estimating the relative pathogenicity of human genetic variants. Nat Genet 2014;46:310-5.

[17] Ritchie GRS, Dunham I, Zeggini E, Flicek P. Functional annotation of noncoding sequence variants. Nat Methods 2014;11:294-6.

[18] Ionita-Laza I, McCallum K, Xu B, Buxbaum JD. A spectral approach integrating functional genomic annotations for coding and noncoding variants. Nat Genet 2016;48:214-20.

[19] Alipanahi B, Delong A, Weirauch MT, Frey BJ. Predicting the sequence specificities of DNA- and RNA-binding proteins by deep learning. Nat Biotechnol 2015;33:831-8.

[20] Zhou J, Troyanskaya OG. Predicting effects of noncoding variants with deep learning-based sequence model. Nat Methods 2015;12:931-4.

[21] Zeng H, Edwards MD, Guo Y, Gifford DK. Accurate eQTL prioritization with an ensemble $\square$ based framework. Hum Mutat 2017;38:1259-65.

[22] Zhou J, Theesfeld CL, Yao K, Chen KM, Wong AK, Troyanskaya OG. Deep learning sequence-based ab initio prediction of variant effects on expression and disease risk. Nat Genet 2018;50:1171-9.

[23] Wilson DJ. The harmonic mean p-value for combining dependent tests. Proc Natl Acad Sci U S A 2019;116:1195-200.

[24] Piñero J, Ramírez-Anguita JM, Saüch-Pitarch J, Ronzano F, Centeno E, Sanz F, et al. The DisGeNET knowledge platform for disease genomics: 2019 update. Nucleic Acids Res 2019;48:D845-55. 
1 [25] Lu Y, Quan C, Chen H, Bo X, Zhang C. 3DSNP: a database for linking human 2 noncoding SNPs to their three-dimensional interacting genes. Nucleic Acids Res 3 2017;45:D643-9.

4 [26] Shi F, Wang Y, Huang D, Liang Y, Liang N, Chen X, et al. Computational

[32] Kalita CA, Brown CD, Freiman A, Isherwood J, Wen X, Pique-Regi R, et al. High-throughput characterization of genetic effects on DNA-protein binding and gene transcription. Genome Res 2018;28:1701-8.

[33] Liu S, Liu Y, Zhang Q, Wu J, Liang J, Yu S, et al. Systematic identification of regulatory variants associated with cancer risk. Genome Biol 2017;18:194.

[34] Doan RN, Bae B, Cubelos B, Chang C, Hossain AA, Al-Saad S, et al. Mutations in human accelerated regions disrupt cognition and social behavior. Cell 2016;167:341-54.

[35] Ulirsch JC, Nandakumar SK, Wang L, Giani FC, Zhang X, Rogov P, et al. Systematic functional dissection of common genetic variation affecting red blood cell traits. Cell 2016;165:1530-45.

[36] Vockley CM, Guo C, Majoros WH, Nodzenski M, Scholtens DM, Hayes MG, et al. Massively parallel quantification of the regulatory effects of noncoding 
1 genetic variation in a human cohort. Genome Res 2015;25:1206-14.

2 [37] Birnbaum RY, Patwardhan RP, Kim MJ, Findlay GM, Martin B, Zhao J, et al.

3 Systematic dissection of coding exons at single nucleotide resolution supports an

4 additional role in cell-specific transcriptional regulation. PLoS Genet

$5 \quad 2014 ; 10: \mathrm{e} 1004592$.

6 [38] Kheradpour P, Ernst J, Melnikov A, Rogov P, Wang L, Zhang X, et al.

7 Systematic dissection of regulatory motifs in 2000 predicted human enhancers

8 using a massively parallel reporter assay. Genome Res 2013;23:800-11.

9 [39] Patwardhan RP, Hiatt JB, Witten DM, Kim MJ, Smith RP, May D, et al.

10 Massively parallel functional dissection of mammalian enhancers in vivo. Nat 11 Biotechnol 2012;30:265-70.

12 [40] Lo SL, Cai CZ, Chen YZ, Chung MC. Effect of training datasets on support 13 vector machine prediction of protein-protein interactions. Proteomics 14 2005;5:876-84.

15 [41] Tomer Y, Huber A. The etiology of autoimmune thyroid disease: A story of 16 genes and environment. J Autoimmun 2009;32:231-9.

17 [42] Fu Y, Tessneer KL, Li C, Gaffney PM. From association to mechanism in 18 complex disease genetics: the role of the 3D genome. Arthritis Res Ther $19 \quad 2018 ; 20: 216$.

20 [43] Kochi Y. Genetics of autoimmune diseases: perspectives from genome-wide association studies. Int Immunol 2016;28:155-61.

[44] Zhu W, He X, Cheng K, Zhang L, Chen D, Wang X, et al. Ankylosing spondylitis: etiology, pathogenesis, and treatments. Bone Res 2019;7:22.

[45] Bossone SA, Asselin C, Patel AJ, Marcu KB. MAZ, a zinc finger protein, binds to $c-M Y C$ and $C 2$ gene sequences regulating transcriptional initiation and termination. Proc Natl Acad Sci U S A 1992;89:7452-6.

[46] Prasad DD, Ouchida M, Lee L, Rao VN, Reddy ES. TLS/FUS fusion domain of TLS/FUS-erg chimeric protein resulting from the $\mathrm{t}(16 ; 21)$ chromosomal translocation in human myeloid leukemia functions as a transcriptional activation domain. Oncogene 1994;9:3717-29.

[47] ENCODE Project Consortium. An integrated encyclopedia of DNA elements in the human genome. Nature 2012;489:57-74.

[48] Musio A, Mariani T, Vezzoni P, Frattini A. Heterogeneous gene distribution reflects human genome complexity as detected at the cytogenetic level. Cancer 
$1 \quad$ Genet Cytogenet 2002;134:168-71.

2 [49] Maan AA, Eales J, Akbarov A, Rowland J, Xu X, Jobling MA, et al. The Y

3 chromosome: a blueprint for men's health? Eur J Hum Genet 2017;25:1181-8.

4 [50] Parker K, Erzurumluoglu AM, Rodriguez S. The Y chromosome: a complex $5 \quad$ locus for genetic analyses of complex human traits. Genes (Basel) 2020;11:1273.

6 [51] Anderson K, Cañadas-Garre M, Chambers R, Maxwell AP, McKnight AJ. The

7 challenges of chromosome $\mathrm{Y}$ analysis and the implications for chronic kidney

8 disease. Front Genet 2019;10:781.

9 [52] Punzi G, Bharadwaj R, Ursini G. Neuroepigenetics of schizophrenia. Prog Mol 10 Biol Transl Sci 2018;158:195-226.

11 [53] Ji X, Dadon DB, Powell BE, Fan ZP, Borges-Rivera D, Shachar S, et al. 3D 12 chromosome regulatory landscape of human pluripotent cells. Cell Stem Cell $132016 ; 18: 262-75$.

14 [54] Benjamini Y, Hochberg Y. Controlling the false discovery rate: a practical and 15 powerful approach to multiple testing. J R Stat Soc Series B Stat Methodol $16 \quad 1995 ; 57: 289-300$.

17 [55] Gel B, Serra E. karyoploteR: an R/Bioconductor package to plot customizable 18 genomes displaying arbitrary data. Bioinformatics 2017;33:3088-90.

19 [56] Guo Y, Tian K, Zeng H, Guo X, Gifford DK. A novel k-mer set memory (KSM) 20 motif representation improves regulatory variant prediction. Genome Res $21 \quad 2018 ; 28: 891-900$.

22 [57] Ernst J, Kellis M. ChromHMM: automating chromatin-state discovery and 23 characterization. Nat Methods 2012;9:215-6. 


\section{$1 \quad$ Figure legends}

\section{Figure 1 Overview of the structure of REVA}

3 Manually curated noncoding variant data as well as supplemental information were

4 stored in the database at two levels: accession and variant data. Accession contained 5 the information about the publication, and variant data contained all related 6 information about the variant. A web interface was built for users to access the data in 7 the database.

\section{Figure 2 Annotation of the variants in REVA}

10 A. The distribution of positive and negative variants in the genome. Mixed region 11 refers to "with more than one type annotation". B. The density distribution of positive 12 and negative variants on chromosomes. A two-sided Fisher's exact test with 13 Benjamini and Hochberg correction [54] was used in the analysis of the chromosome 14 distribution of variants. The cutoff for the adjusted p-value was set to 0.05. The density distribution plot was constructed with the karyoploteR package [55] in R. No variants were located on the Y chromosome.

Figure 3 Performance of involved tools on the benchmarking dataset

A. The performance comparison of involved tools and bubbles colored by F1 score. The results are ordered by F1 score. B. The ROC curves for involved tools. C.

21 Performance comparison of the involved tools except EnsembleExpr on variants that 22 were also included in GWAS catalog and not. D. Performance comparison of involved 23 tools except EnsembleExpr on variants that had different phastCons100way scores. E. 24 Performance comparison of involved tools except EnsembleExpr on variants from different cell lines. "All" represents the F1 score in Figure 3A. F. Performance comparison of involved tools except EnsembleExpr on variants that were also included in HGMD. "All" represents the F1 score in Figure 3A. "All HGMD" represents the F1 score on all variants that were included in HGMD. "DM?", "DP", "FP", and "DFP" refer to the classes of related variants documented in HGMD.

\section{Figure 4 Illustration of the Web interface of REVA}

32 A. Chromatin profile feature plot in "Annotation" module of variant detail page. 33 Chromatin features are presented by category. Users can hover the mouse over the 
1 outlier or the box to show more information. At the right of boxplot is a table to show 2 detail information. Users can click the boxplot to show corresponding category. $\mathbf{B}$. 3 Chromatin profile feature heatmap in "Annotation" module of variant detail page. The 4 heatmap is presented by cell line and each row in heatmap corresponding to one 5 category. Users can click the cell line/tissue list at the right of heatmap to render

6 annotation in target cell line/tissue and hover the mouse over the block in heatmap to 7 show feature information. Both Figure $4 \mathrm{~A}$ and $4 \mathrm{~B}$ are retrieved from 8 http://reva.gao-lab.org/detail.php?id=intid1_8498680_8438620atk562\&reference=GR

9 Ch38.

10

11 Tables

12 Table 1 Properties of involved computational tools

13 Table 2 Variant information extracted during the data collection process 14 


\section{Supplementary material}

\section{Figure S1 Database scheme of REVA}

3 All data were stored in MongoDB with five collections. "data_experiment_info" 4 contained accession information and "data_experiment" contained variant data. The 5 original variant data included in meta-analysis stored in "data_experiment_meta". 6 “data_experiment_anno" and "disease_variant" contained variant annotation and 7 associated diseases and phenotypes.

Figure S2 Annotation of the variants in REVA

A. The ratio and number of positive and negative variants that have $0,1,2,3$ or 4 categories of annotations.

B. Average ratio of annotations of variants by category.

13 Figure S3 Performance comparison of involved tools except EnsembleExpr on variants that were also included in ClinVar

"All" represents the F1 score in Figure 3A. "All ClinVar" represents the F1 score on all variants that were included in ClinVar. "Benign", "Likely benign", and "Drug response" refer to the clinical significance of related variants documented in ClinVar.

\section{Figure S4 Illustration of the web interface of REVA}

A. Advanced search page (http://reva.gao-lab.org/advanced_search.php). Users can conduct customized search or batch search through this page. B. Search results page. Users can sort searching results by "Chr", "Pos", "Label”, "Effect", "Fragment Active/Repress", "Adjusted P Value", or "Cell Line".

Figure S5 The combined search result of "ankylosing spondylitis"

A. Searching result sorted by "Effect". Variant rs4456788 has the largest effect size in rs4456788. This variant is also considered to repress gene expression in K562 cell line. C. The chromatin profile feature plot of rs4456788 focusing on transcription

30 factor binding in HepG2 cell line, sorted by $\log 2$ fold change. The variant is

31 considered to decrease the binding affinity of transcription factors MAZ and FUS. 
1 Protocol details were extracted from original literature and as an attachment in the

2 accession (http://reva.gao-lab.org/download/protocol/reva-0002.pdf).

3

4 Tables

5 Table S1 Thresholds of published state-of-the-art tools that we evaluated

6 Table S2 Distribution of locus of positive and negative variants

7 Table S3 Distribution of positive and negative variants on chromosomes

8 Table S4 Annotations of variants with 689 ENCODE profiles

9 Table S5 Information of all ENCODE profiles

10 Table S6 Performance of involved tools on the benchmarking dataset

11 Table S7 Cell line information of benchmark dataset

12 Table S8 Variants in benchmark dataset overlapped with HGMD

13 Table S9 Variants in benchmark dataset overlapped with ClinVar

14 


\section{Search publications}

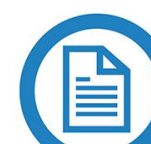

Manually examine

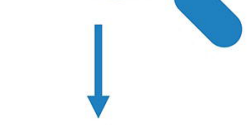

Accession

number

Created time

Last updated time

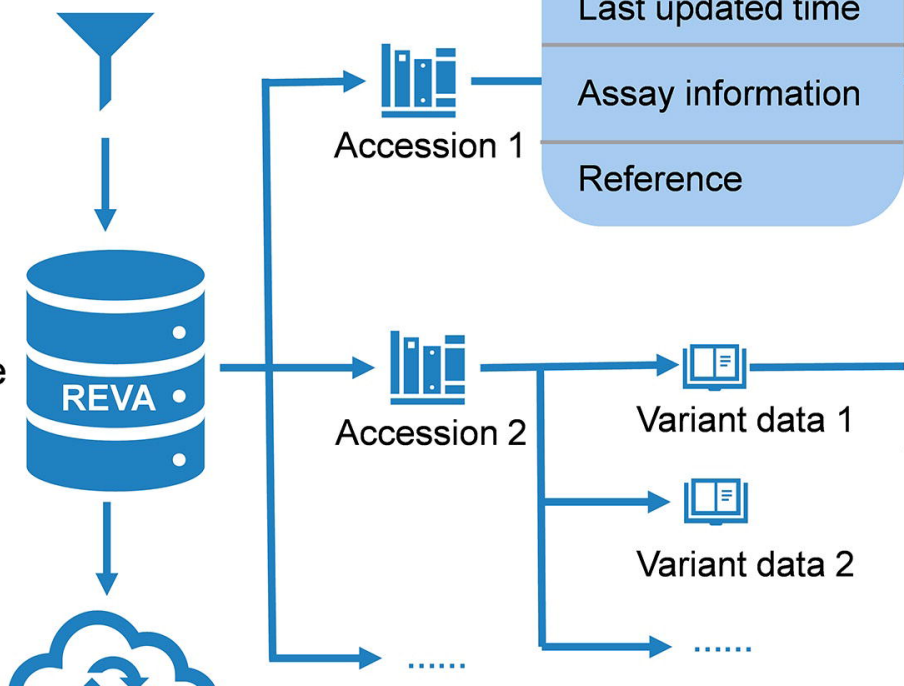

Load into database

Web server

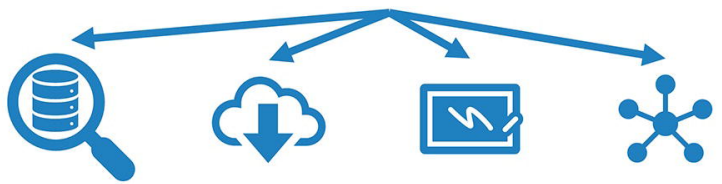

Search Download Annotation Benchmark
Genome location Reference SNP id Reference allele Alternative allele

Label

Raw p-value Adjusted $\mathrm{p}$-value Effect size

Fragment effect

Experimental cell line Genomic region TF

TF effect 
Intron

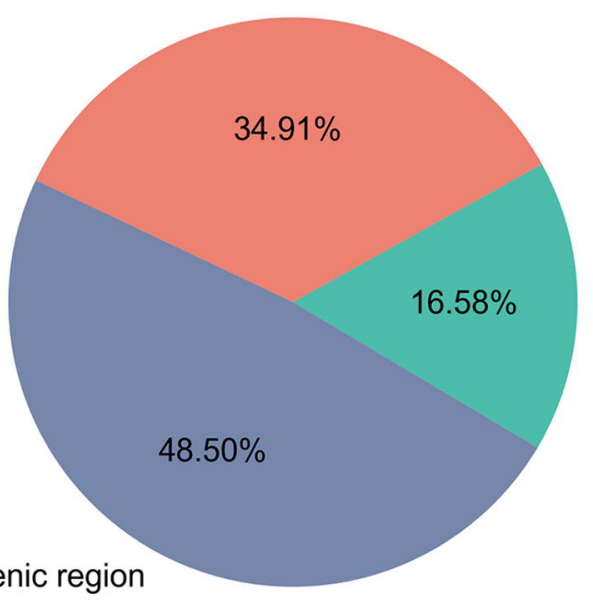

Intron

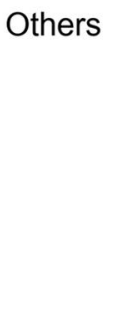

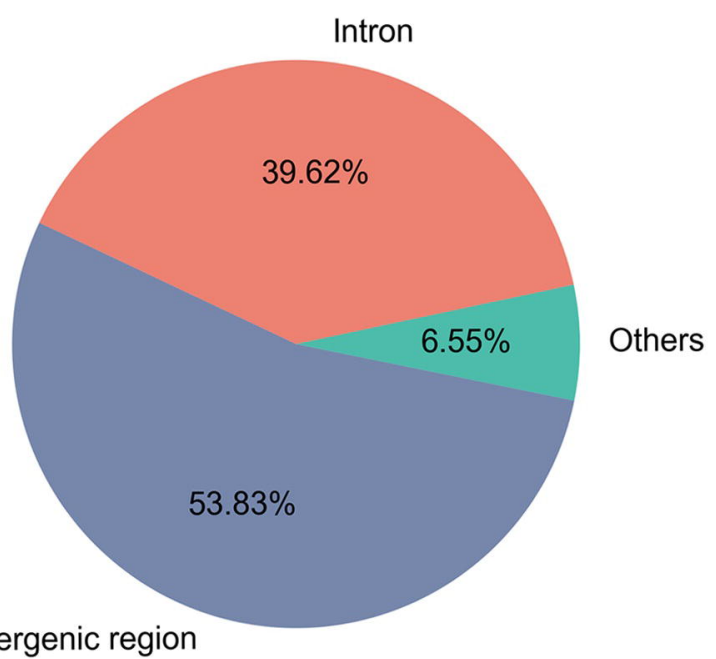

B

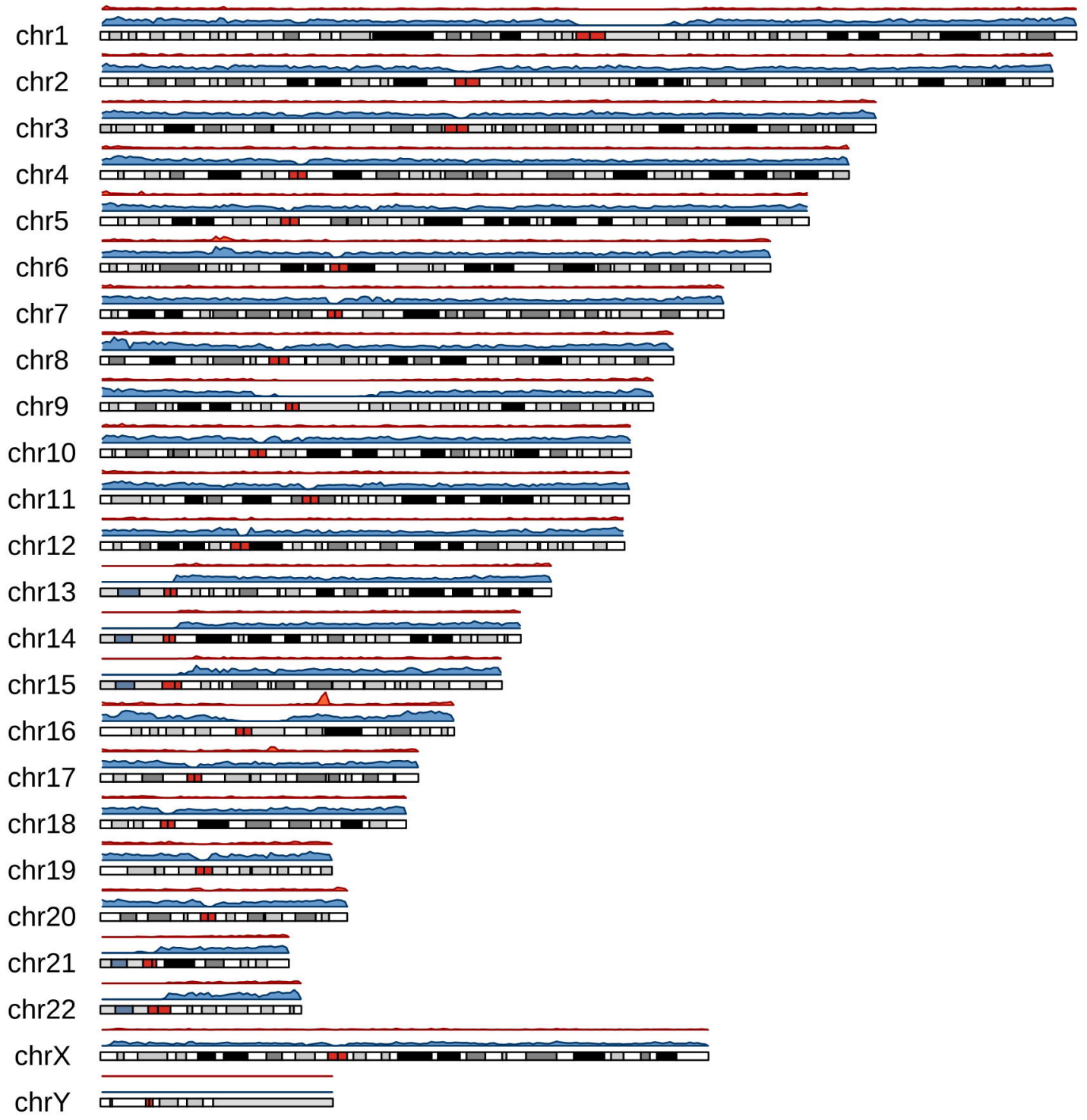




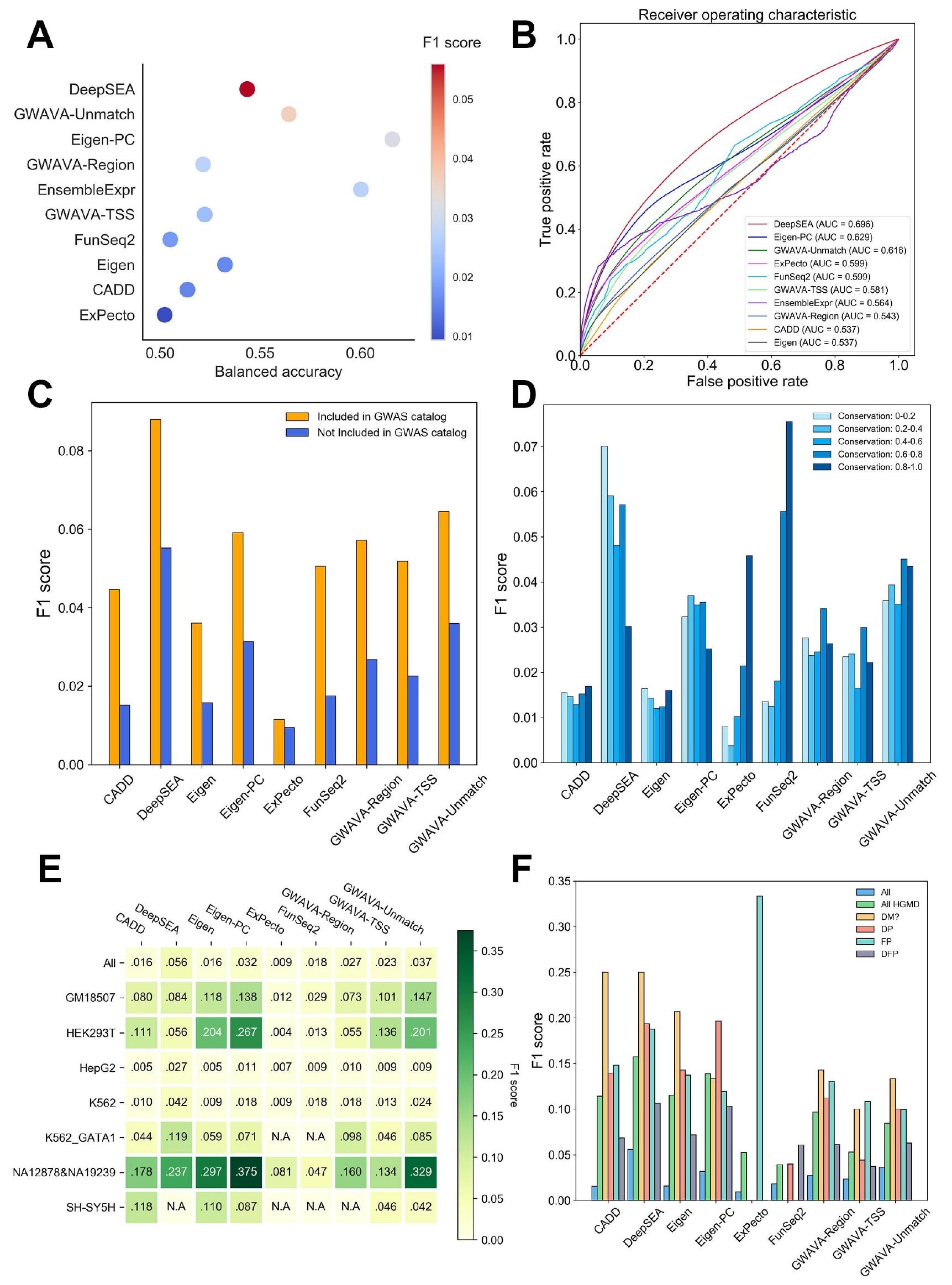


A Log fold change

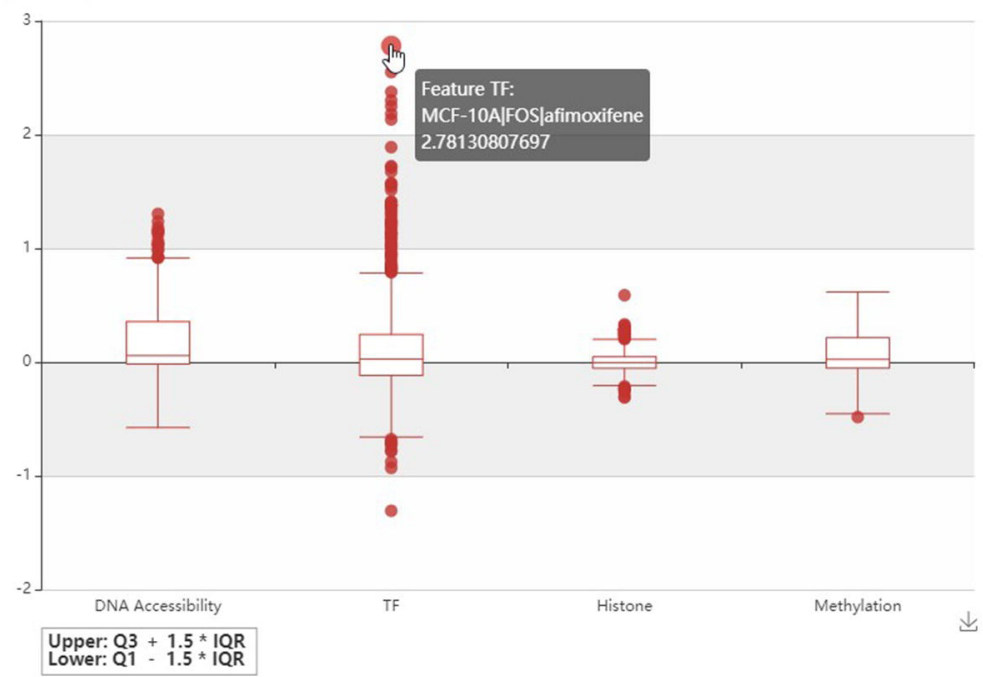

B Lower: Q1 - $1.5 *$ IQR
DNA Accessibility

Search:

\begin{tabular}{|c|c|c|c|c|c|c|c|}
\hline $\begin{array}{l}\text { Cell line / } \\
\text { Tissue }\end{array}$ & \multicolumn{2}{|c|}{ Feature } & $\uparrow \downarrow$ & \multicolumn{4}{|c|}{ Log fold change } \\
\hline $8988 \mathrm{~T}$ & \multicolumn{3}{|c|}{ DNase-seq } & \multicolumn{4}{|c|}{-0.142641733985} \\
\hline A172 & \multicolumn{3}{|c|}{ DNase-seq } & \multicolumn{4}{|c|}{0.757326313713} \\
\hline A673 & \multicolumn{3}{|c|}{ DNase-seq } & \multicolumn{4}{|c|}{0.0799953200128} \\
\hline $\mathrm{ACHN}$ & \multicolumn{3}{|c|}{ DNase-seq } & \multicolumn{4}{|c|}{0.2822895176} \\
\hline Adipocyte & \multicolumn{3}{|c|}{ DNase-seq } & \multicolumn{4}{|c|}{0.433284923105} \\
\hline Adrenal gland & \multicolumn{3}{|c|}{ DNase-seq } & \multicolumn{4}{|c|}{-0.0152639004104} \\
\hline Previous & 1 & 3 & 4 & 5 & $\ldots$ & 47 & Next \\
\hline
\end{tabular}

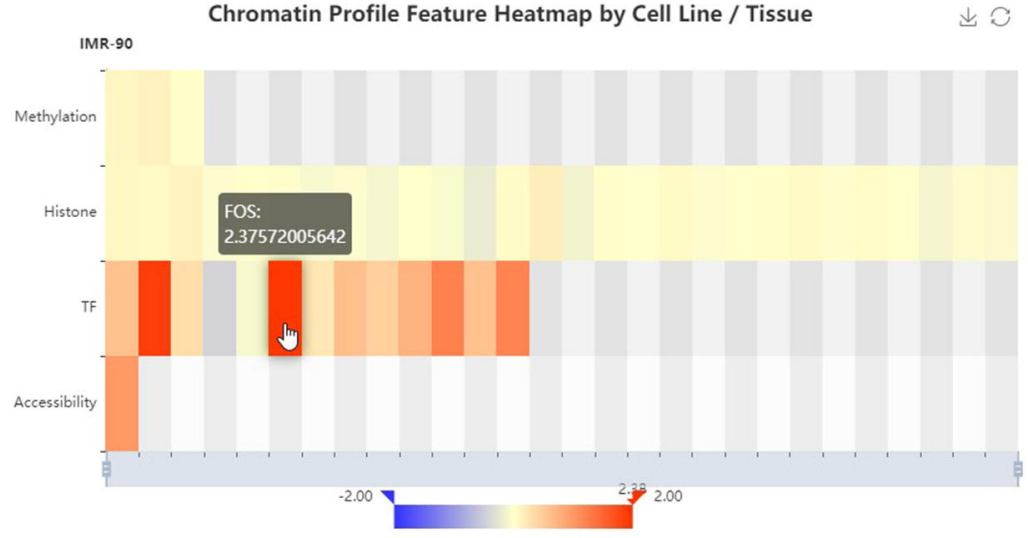

IMR-90

Chromatin Profile Feature Heatmap by Cell Line / Tissue

$\curvearrowright$

Cell line / Tissue

HTR-8/SVneo

HUES48

HUES 6

HUES64

HuH 7

HuH-7.5

IMR-90

\begin{tabular}{|l|l|l|l|l|l|l|l|l|}
\hline Previous & 1 & $\ldots$ & 20 & 21 & 22 & $\ldots$ & 44 & Next \\
\hline
\end{tabular} 
Table 1 Properties of involved computational tools

\begin{tabular}{|c|c|c|c|}
\hline Tool & $\begin{array}{l}\text { Modeling } \\
\text { Approach }\end{array}$ & Model Features & Output \\
\hline FunSeq2 & Knowledge-based & $\begin{array}{l}\text { Evolutionary parameters; } \\
\text { Encode summaries; PWMs; } \\
\text { likely target genes; biological } \\
\text { networks; recurrent elements } \\
\text { across cancer samples }\end{array}$ & Cancer driver mutations \\
\hline CADD & $\begin{array}{l}\text { Supervised } \\
\text { learning }\end{array}$ & $\begin{array}{l}\text { Evolutionary parameters; } \\
\text { Encode summaries; } \\
\text { population frequencies; } \\
\text { transcript information; } \\
\text { protein-level scores }\end{array}$ & Functional variants \\
\hline GWAVA & $\begin{array}{l}\text { Supervised } \\
\text { learning }\end{array}$ & $\begin{array}{l}\text { Evolutionary parameters; } \\
\text { Encode summaries; } \\
\text { population frequencies }\end{array}$ & Diseased-related variants \\
\hline Eigen & $\begin{array}{l}\text { Unsupervised } \\
\text { learning }\end{array}$ & $\begin{array}{l}\text { Evolutionary parameters; } \\
\text { Encode summaries; } \\
\text { population frequencies }\end{array}$ & Functional variants \\
\hline DeepSEA & $\begin{array}{l}\text { Supervised } \\
\text { learning (deep } \\
\text { learning) }\end{array}$ & $\begin{array}{l}\text { Local sequences; } \\
\text { Evolutionary parameters }\end{array}$ & Functional variants \\
\hline EnsembleExpr & Ensemble-based & $\begin{array}{l}\text { Including features used by } \\
\text { DeepSEA, DeepBind, KSM } \\
\text { [56] and ChromHMM [57] }\end{array}$ & $\begin{array}{l}\text { Expression-modulating } \\
\text { variants }\end{array}$ \\
\hline ExPecto & $\begin{array}{l}\text { Supervised } \\
\text { learning (deep } \\
\text { learning) }\end{array}$ & Local sequences & $\begin{array}{l}\text { Expression-modulating } \\
\text { variants }\end{array}$ \\
\hline
\end{tabular}


1 Table 2 Variant information extracted during the data collection process

\begin{tabular}{|c|c|}
\hline Information & Note \\
\hline Genome location & $\begin{array}{l}\text { Genome location of the variant. Strand } \\
\text { information is also included. For variants from } \\
\text { human, the reference genome was converted to } \\
\text { both GRCh37 and GRCh38. }\end{array}$ \\
\hline Reference SNP id & Reference SNP id of the variant. \\
\hline Reference allele & Reference allele of the variant. \\
\hline Alternative allele & Alternative allele of the variant. \\
\hline Raw p-value & Raw p-value given by the publication. \\
\hline Adjusted p-value & $\begin{array}{l}\text { If the publication did not provide adjusted } \\
\text { p-value, the method of Benjamini and Hochberg } \\
\text { was conducted. }\end{array}$ \\
\hline Cutoff & $\begin{array}{l}\text { The cutoff for the adjusted p-value. If the } \\
\text { publication did not provide a cutoff, the cutoff } \\
\text { was set to } 0.05 \text {. }\end{array}$ \\
\hline Label & $\begin{array}{l}\text { Given based on the cutoff for the adjusted } \\
\text { p-value provided in the publication. If the } \\
\text { adjusted p-value was less than the cutoff, the } \\
\text { label would be } 1 \text {, otherwise, the label would be } 0 \text {. }\end{array}$ \\
\hline Effect size & Effect size provided by the publication. \\
\hline Fragment effect & $\begin{array}{l}\text { The effect of the fragment carrying the variant, } \\
\text { given based on the effect size: activation, } \\
\text { repression or no effect. }\end{array}$ \\
\hline Experimental cell line & The cell line used to conduct the experiment. \\
\hline Genomic region & $\begin{array}{l}\text { The genomic region in which the variant is } \\
\text { located, such as the particular gene and intron. }\end{array}$ \\
\hline $\mathrm{TF}$ & Transcription factor related to the variant. \\
\hline TF effect & $\begin{array}{l}\text { The effect of the above TF: activation or } \\
\text { repression. }\end{array}$ \\
\hline
\end{tabular}




\section{data_experiment_meta}

disease_variant

Inter_id

Meta_id

$\mathrm{Chr}$

Pos_37

Pos_38

Rs

Ref

Alt

Start

End

Direction

Region

TF

Cell_line

$P$ value

FDיR

Effect

Variant_active_repress

Label

TF_active_repress

Accession_number

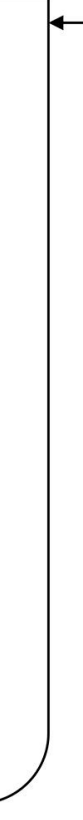

\section{data_experiment_anno}

Anno_id

Annotation

\section{data_experiment}

Inter_id

Anno-id

Chr

Pos_37

Pos_38

Rs

Ref

Alt

Start

End

Direction

Region

TF

Cell_line

P_value

FDיR

Effect

Variant_active_repre ss

Label

TF_active_repress

Accession_number snpld

Chromosome

Position

DSI

DPI

diseaseld

diseaseName

diseaseType

diseaseClass

diseaseSemanticType

Score

EI

Yearlnitial

YearFinal

NofPmids

source

data_experiment_info

Accession_number

Created_time

Updated_time

Method_category

Method_detail

Raw_data

Species

Reference_genome_vers

ion

Cutoff

Literature

Doi 
A
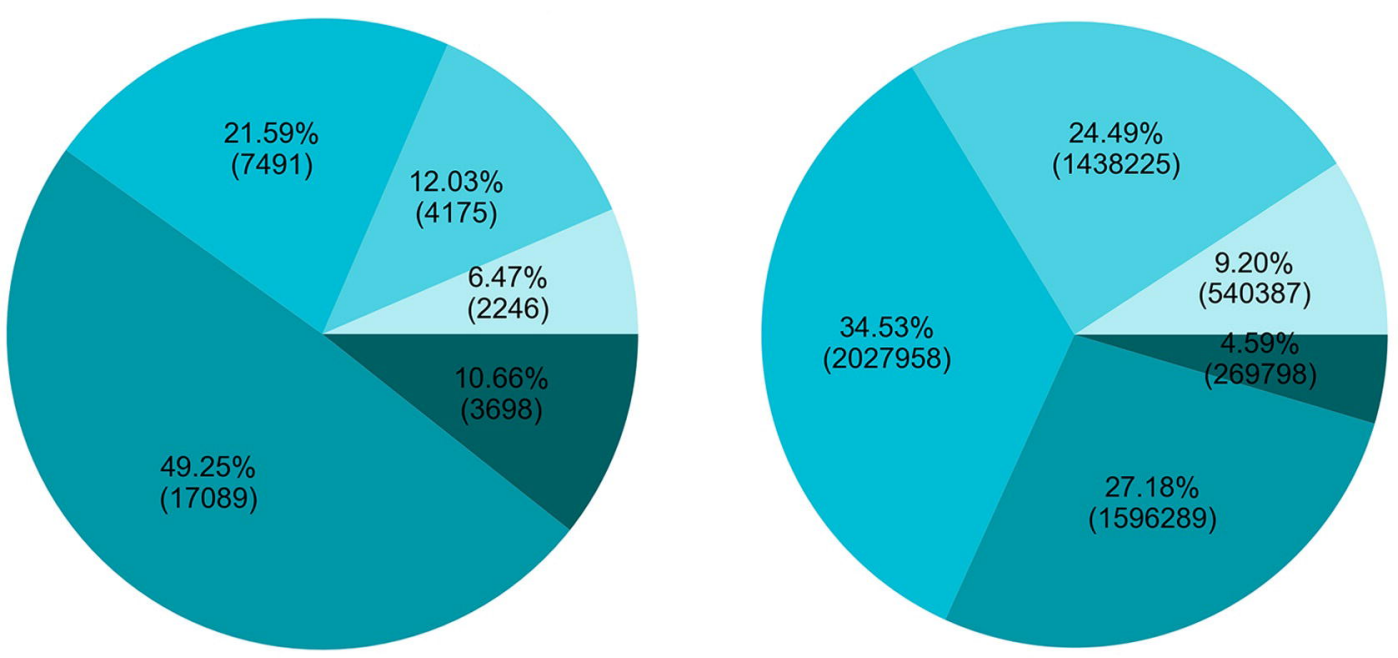

Number of annotation categories

0

1
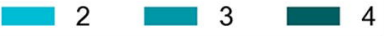

B
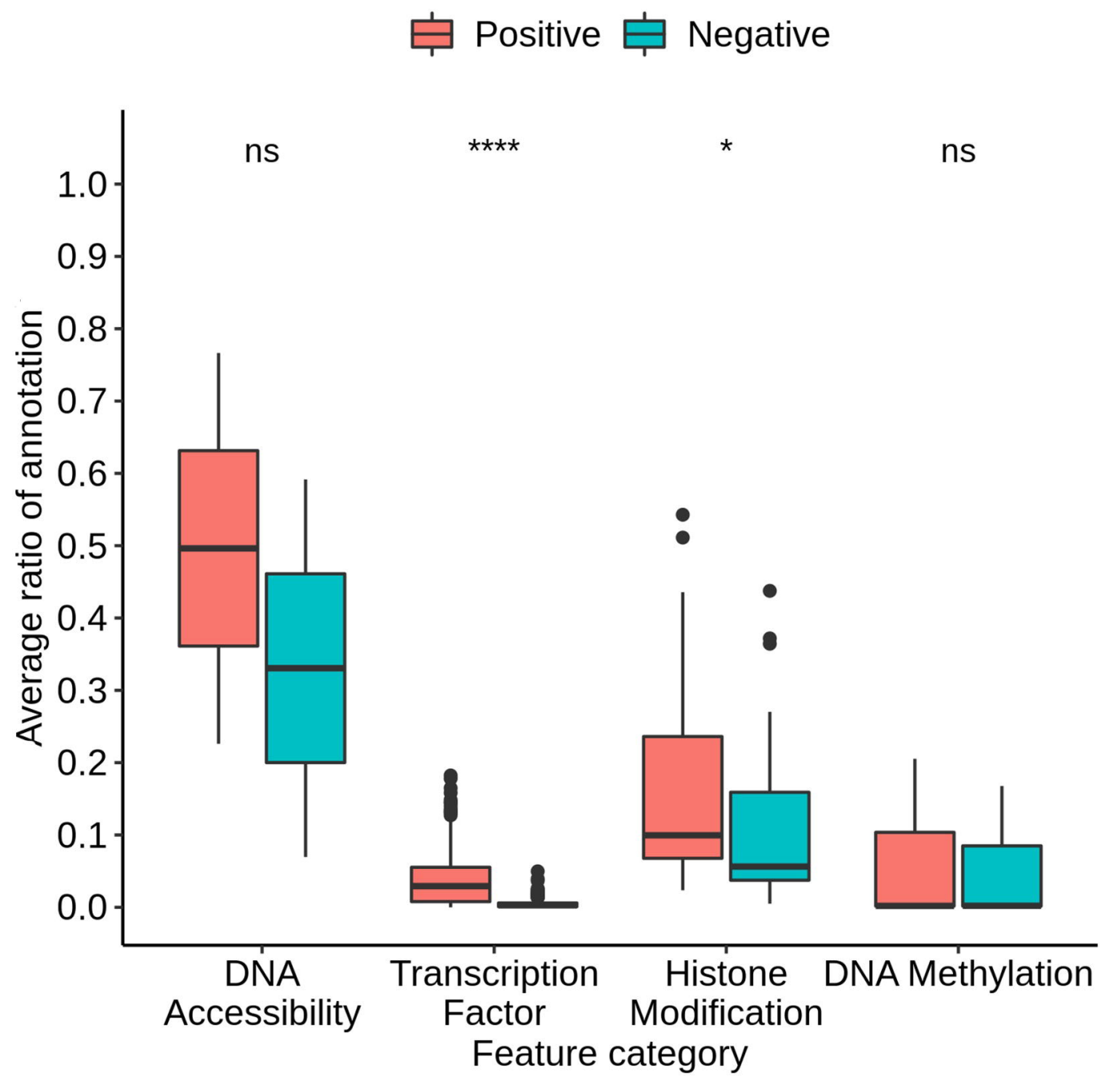


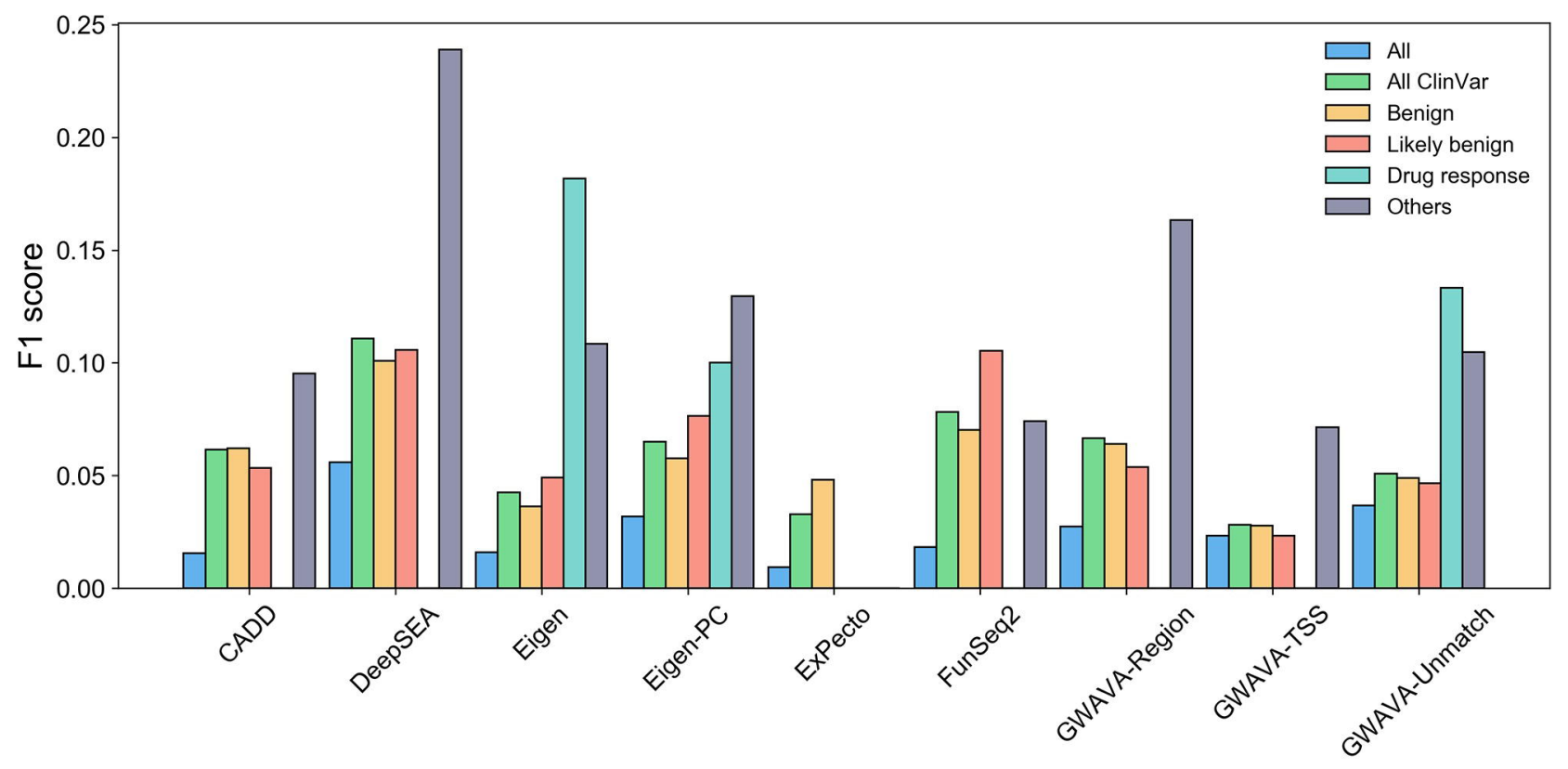




\section{Q Combined Search}

\section{Genomic Assembly:}

\section{GRCh38 $\vee$ GRCh37}

Label:

$\checkmark$ Positive $\checkmark$ Negative

Cell line: All selected

Gene search range: 1000

\section{Q Batch Search}

Please paste below to query variants.

The maximum number of your queries is 100 .

\section{Or upload the file:}

选择文件 未选择任何文件

\section{(2) Notes}

Combined search allow you search variants with specific genomic assembly, label and cell line.

Query: chromosome with position range, rs id, gene name, ensembl gene id and disease name. You can click demo bellow if necessary. Gene search range is functioned only with gene name or ensembl gene id as the input.

Example: demo\#1 demo\#2

\section{Search Q}

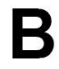

\section{Q Combined Search}

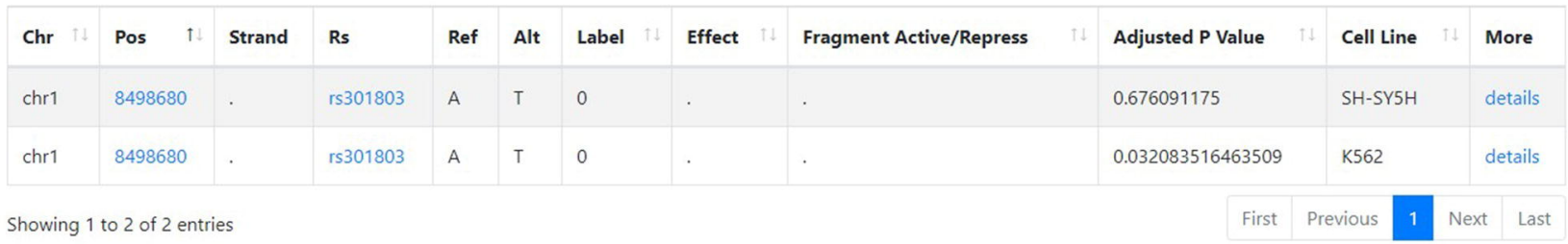




\begin{tabular}{|c|c|c|c|c|c|c|c|c|c|c|c|}
\hline Chr $1 \downarrow$ & Pos $\quad 11$ & Strand & Rs & Ref & Alt & Label 11 & Effect & Fragment Active/Repress & Adjusted P Value & Cell Line & More \\
\hline chr21 & 45616324 & . & rs4456788 & G & A & 1 & -1.91441950595 & Repress & . & HepG2 & details \\
\hline chr8 & 129567181 & . & rs6651252 & $\mathrm{T}$ & $\mathrm{C}$ & 1 & -1.87468724523 & Repress & . & HepG2 & details \\
\hline chr1 & 154426264 & . & rs4129267 & C & $\mathrm{T}$ & 1 & -1.47937165102 & Repress & . & HepG2 & details \\
\hline chr21 & 45616324 & . & rs4456788 & G & A & 1 & -1.29400001917 & Repress & . & K562 & details \\
\hline chr9 & 117553249 & . & rs 4246905 & $T$ & C & 1 & -0.754254696209 & Repress & . & K562 & details \\
\hline chr10 & 90749963 & . & rs1800682 & A & G & 1 & 0.270957373 & Active & 0.0000332 & HEK293T & details \\
\hline chr10 & 64438486 & . & rs10995271 & G & C & 1 & 0.744397932203 & Active & . & HepG2 & details \\
\hline chr11 & 65653242 & - & rs568617 & C & $\mathrm{T}$ & 1 & . & . & 1.863862332 & NA12878, NA19239 & details \\
\hline Showing & 08 of 8 ent & & & & & & & & First & Previous & Last \\
\hline
\end{tabular}

\section{B}

\section{$O$ Cell Line and Expression}

\begin{tabular}{|c|c|c|c|c|c|c|}
\hline Cell Line & Effect & Adjusted P Vaule & Label & Fragment Active/Repress & TF & TF Active/Repress \\
\hline HepG2 & -1.91441950595 & . & 1 & Repress & . & . \\
\hline K562 & -1.29400001917 & . & 1 & Repress & . & . \\
\hline
\end{tabular}

\section{C}

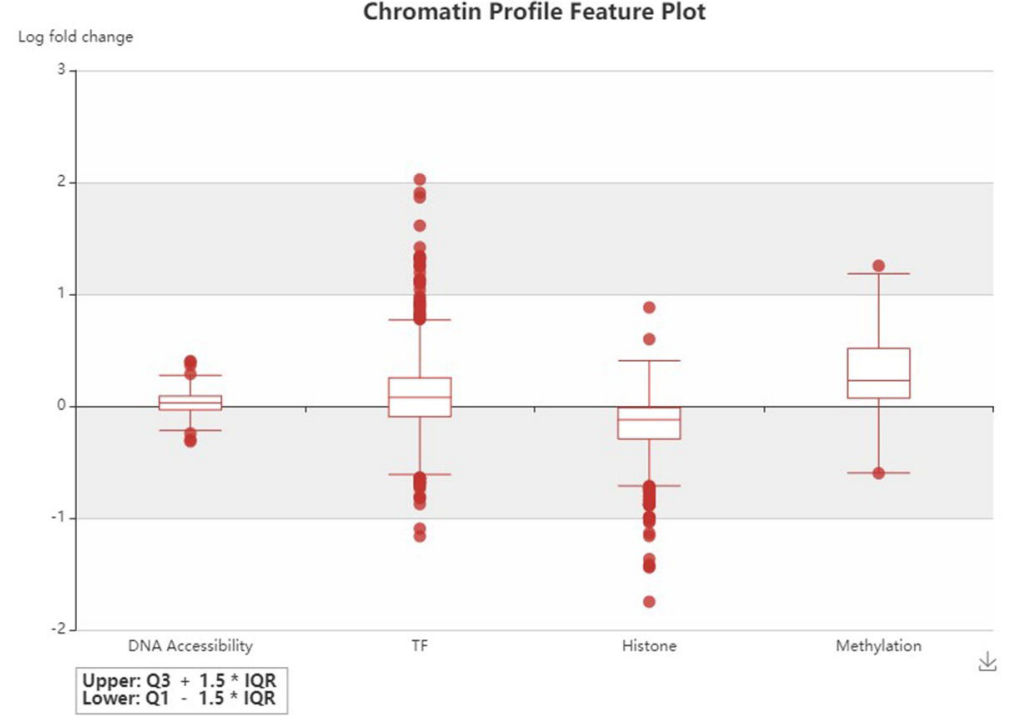

TF

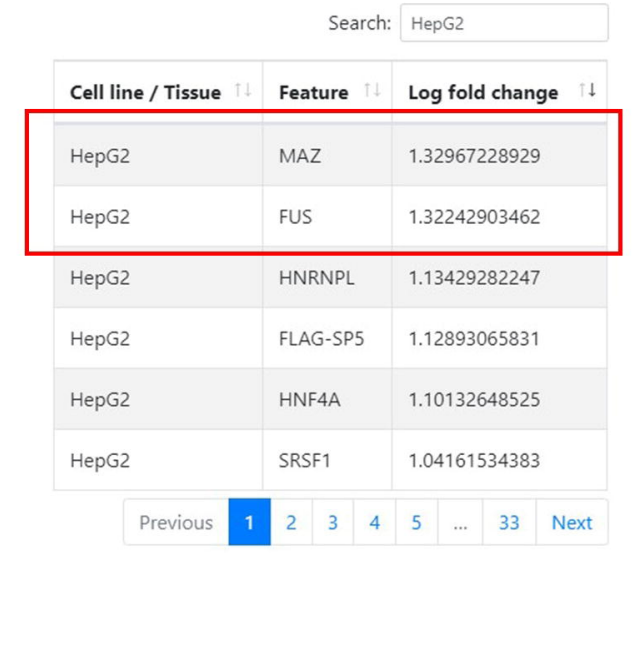


Oligonucleotide library design and synthesis

Oligonucleotide libraries were designed to contain, in order, the universal primer site ACTGGCCGCTTCACTG, the variable $145 \mathrm{bp}$ test sequence, $\mathrm{KpnI} / \mathrm{XbaI}$ restriction sites (GGTACCTCTAGA), a variable 10-bp tag sequence, and the universal primer site AG ATCGGAAGAGCGTCG. Each sequence was tested with 10 unique tags in order to reduce variance due to stochastic rates of amplification of specific plasmids. If a putative enhancer or any of its manipulations contained the recognition sequence for any restriction enzyme (GGTACC, TCTAGA, or GGCCNNNNNGGCC), then that putative enhancer was excluded and an additional one was chosen. The resulting 54,000-plex 200-mer oligonucleotide libraries were synthesized by Agilent, Inc.

\section{MPRA plasmid construction}

Full-length oligonucleotides were isolated using 10\% TBE-Urea polyacrylamide gel (Invitrogen) and then amplified by $20-26$ cycles of emulsion PCR using Herculase II Fusion DNA Polymerase (Agilent) and primers containing SfiI sites. Purified PCR products were then digested with SfiI (NEB) and directionally cloned into the SfiIdigested MPRA vector pGL4.10M using One Shot TOP10 Electrocomp E. coli cells (Invitrogen). To preserve library complexity, the efficiency of transformation was maintained at $>3 \times 10^{8} \mathrm{cfu} / \mathrm{mg}$. The isolated plasmid pool was digested with $\mathrm{KpnI} / \mathrm{Xbal}$ to cut between the tested sequence and tag, ligated with a synthetic KpnI-Xbal fragment containing the SV40 early enhancer/promoter (derived from pGL4.73, Promega) and the luc2 luciferase ORF (derived from pGL4.10, Promega) and then transformed into E. coli as described above. Finally, to remove the vector background, the resultant plasmid pool was digested with KpnI, size selected on a $1 \%$ agarose gel, self-ligated and retransformed into $E$. coli.

\section{Cell culture and transfection}

HepG2 cells (ATCC HB-8065) were maintained in Eagle's Minimum Essential Medium supplemented with $10 \%$ fetal bovine serum (FBS) penicillin $(50$ units $/ \mathrm{mL})$ and streptomycin $(50 \mu \mathrm{g} / \mathrm{mL})$. For HepG2 transfections, $5 \times 10^{6}$ cells were plated in $15-\mathrm{cm}$ plates. Transfections were performed $24 \mathrm{~h}$ after plating using Fugene HD (Promega) according to the manufacturer's instructions. In each transfection we used $15 \mu \mathrm{g}$ of DNA and a Fugene:DNA ratio of 7:2. K562 cells (ATCC CCL-243) were cultured in RPMI-1640 supplemented with $10 \%$ FBS and $1 \%$ GIBCO Antibiotic-Antimycotic (Invitrogen). For K562 transfections, $20 \mathrm{mg}$ of DNA was introduced into $4 \times 10^{6}$ cells using a Nucleofector II device with Nuclefector Kit Vand program T-016; 24 h posttransfection/nucleofection, cells were lysed in RLT buffer (Qiagen) and frozen at $-80^{\circ} \mathrm{C}$. Total RNA was isolated from cell lysates using RNeasy kit (Qiagen). We chose the transfection method for each cell line that maximized efficiency while minimizing cell death.

\section{Tag-seq}

mRNA was extracted from $100 \mu \mathrm{g}$ of total RNA using MicroPoly(A)Purist kits (Ambion) and treated with DNase I using the Turbo DNA-free kit (Ambion). First- strand cDNA was synthesized from 400 to 700 ng of mRNA using the High Capacity RNA-to-cDNA kit (Applied Biosystems). Tag-seq sequencing libraries were generated directly from $10 \%$ of a cDNA reaction or $50 \mathrm{ng}$ of plasmid DNA by 26 cycle PCR using Pfu Ultra II HS DNA polymerase $2 \mathrm{X}$ master mix (Agilent) and primers. The resultant PCR products were size-selected using $2 \%$ agarose E-Gel EX (Invitrogen). The libraries were sequenced in indexed pools of eight or individually using 36-nt singleend reads on an Illumina HiSeq 2000 instrument.

\section{Data processing and normalization}

To infer the tag copy numbers in each Tag-seq library, all sequence reads were examined, regardless of their quality scores. If the first 10 nucleotides of a read perfectly matched one of the 54,000 designed tags, and the remaining nucleotides matched the expected upstream MPRA construct sequence, this was counted as one occurrence of that tag. All reads that did not meet this criterion were discarded. This procedure was repeated separately for the plasmid, HepG2 mRNA, and K562 mRNA pools. The plasmid and mRNA counts for each tag was normalized by the total number of counts from the respective source, and a ratio of the mRNA to plasmid counts was then generated for each tag. A single value was produced for each tested sequence by taking the mean over the tags/replicates, excluding any that had fewer than 40 plasmid reads. The $\log _{2}$ of this value divided by the median was used throughout (this normalization is monotonic and consequently does not affect the P-values for the statistical tests used). Because only a small portion of our tested sequences corresponded to what we later determined to be a functional wild-type enhancer or a nondisruptive mutation, we estimate the 0 baseline level to be approximately the background level of expression for our promoter. Consistent with this, the 2098 sequences with scrambled motifs (and thus no expected expression) have a mean normalized expression of -0.0054 for HepG2 cells and -0.06 for K562 cells. Five probes had 0 RNA counts and their $\log _{2}$ values were replaced by 7 (the smallest non-zero mean had a $\log _{2}$ of -6.82 ).

\section{Statistical analysis}

The paired Wilcoxon signed-rank test is used for comparing different versions of the same set of sequences (e.g., original to scramble). The unpaired Mann-Whitney U-test is used to compare two different sets of sequences (e.g., conserved versus ignoring conservation). Combined P-values are calculated, when indicated, by taking the expression values across multiple factors and using them together for the corresponding statistical test by treating them as one list of values. Where replicates for two sequences are directly compared, we use the individual log replicate values with the unpaired, unequal variance Student's t-test. Correlations are computed using Pearson's r, and corresponding permutation P-values are computed as the percentile of the absolute correlation amongst 10 million absolute correlations between the vectors randomly shuffled. P-values are computed in a two-tailed manner, unless otherwise specified. 